\title{
Modeling the Equilibrium Bus Line Choice Behavior and Transit System Design with Oblivious Users
}

\author{
Chuan-Lin Zhao and Hai-Jun Huang \\ School of Economics and Management, Beihang University, Beijing 100191, China \\ Correspondence should be addressed to Chuan-Lin Zhao; chelone@163.com \\ Received 15 November 2013; Accepted 1 January 2014; Published 6 February 2014 \\ Academic Editor: Huimin Niu
}

Copyright ( 2014 C.-L. Zhao and H.-J. Huang. This is an open access article distributed under the Creative Commons Attribution License, which permits unrestricted use, distribution, and reproduction in any medium, provided the original work is properly cited.

In most of transportation literature, users are assumed to be perfectly rational in minimizing their own travel costs or perceived travel costs. However, users may not be perfectly rational in implementing their choices in reality. There exists a kind of boundedly rational users, that is, oblivious users. These oblivious users make their route choices by simple criteria, for example, selecting the shortest (or the most direct) route only based on physical distance or simply following routes recommended by a GPS system. This paper investigates how the existence of oblivious users affects the equilibrium bus line choice behavior in a public transit system. And we propose a method to design a more realistic system.

\section{Introduction}

The purpose of this paper is duple to advance our understanding on the boundedly rational behavior of public transit users when choosing bus lines in a transit network and to design a more realistic public transit system when considering the boundedly rational users.

In the literature, user equilibrium models play an important role in the traffic assignment problems. By assuming that all road users behave in a completely rational way and seeking to minimize their own disutility, Wardrop [1] defined a state of route choice, the so-called user equilibrium (UE). At the UE state, no user can further improve her or his utility by unilaterally changing routes. By relaxing some of the behavioral restrictions implied in a strict deterministic disutility minimization rule, Daganzo and Sheffi [2] developed a stochastic user equilibrium (SUE) model that considers the travelers' imperfect perceptions of travel times. The SUE is achieved when users can no longer change their perceived utility. Existence and uniqueness of UE or SUE in general networks have been well investigated in the literature, including the solution methods for obtaining these two states; see Sheffi [3] and Yang and Huang [4] for more details.

The third equilibrium type is boundedly rational user equilibrium (BRUE). As a relaxation of perfect rationality and optimality, the notion of bounded rationality was proposed by Simon [5] and introduced to traffic modeling by Mahmassani and Chang [6]. It has been shown that bounded rationality is important in many contexts (see, e.g., Conlisk [7] and references cited therein). In the transportation field, Mahmassani and Chang [6] studied the existence, uniqueness, and stability properties of BRUE in the standard singlelink bottleneck network. Many simulation and experimental studies have incorporated the travelers' boundedly rational behavior (e.g., $\mathrm{Hu}$ and Mahmassani [8], Mahmassani and Liu [9], and Mahmassani [10]). Lou et al. [11] are the first to systematically examine the mathematical properties of BRUE in a network traffic assignment context. More specifically, as reported in Mahmassani and Chang [6] and discussed by Lou et al. [11] and Di et al. [12], BRUE flow distributions in a static network may not be unique and the set of all possible BRUE flow distributions is a nonconvex and nonempty set.

Recently, Karakostas et al. [13] extended the traditional UE models by considering one kind of boundedly rational users, that is, oblivious users. These users decide their routing only according to the shortest paths observed on a map.

The above studies are only subject to private car systems. In this paper, we will proceed to our study in a public transit system. In the microeconomic analysis of urban public transportation, two types of resources have to be taken into 


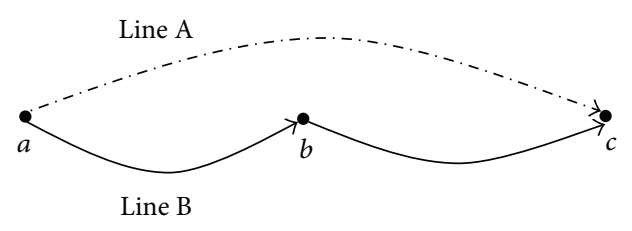

FIGURE 1: A transit network with two lines.

account: those provided by operators, such as vehicles, fuel, terminals, or labor, and those provided by users, namely, their time, usually divided into waiting, access for, and in-vehicle times. In addition, Kraus [14], Lam et al. [15], and Huang et al. [16] introduced the concept of body crowding cost which is related to the passenger/capacity ratio. After Vickrey's view [17], Mohring [18] constructed a microeconomic model to determine optimal frequency of buses serving a corridor with fixed demand. The main result was that frequency should be proportional to the square root of demand, and this happened only because all resources (operators' and users' cost) were considered when finding the minimum cost operation. JaraDíaz et al. [19] analyzed and compared the total value of the resources consumed (operators' and users' time) under four line structures. The role of users' costs was shown to be crucial. This approach has evolved along the last decades, improving our understanding on public transport operations. These studies are based on a strict hypothesis that all users are perfectly rational. In reality, however, most of the users may be boundedly rational in choosing the bus lines.

There is a kind of users, called oblivious users, who make their bus lane choices without caring about the delay and in-carriage congestion experienced. Their decisions rely on simple criteria, for example, finding the most direct line from the transit map. Recently, Raveau et al. [20], through analyzing actual data, verified such an observation that between two routes with identical trip users prefer the most direct route to indirect one with transfer. They also found that the perceptions of transport users regarding available route alternatives are such that they do not always choose what the modeler would consider as the "lowest cost" option. Inspired by this finding, we are naturally interested in the following questions: how the existence of oblivious users affects the equilibrium bus line choice behavior in public transit system and how to design a more realistic transit system when oblivious users exist.

The remainder of this paper is organized as follows. In Section 2, we model the equilibrium bus line choice behavior in a transit network with oblivious users. In Section 3, considering the design variable, that is, bus frequency, we design the system. Section 4 concludes the paper.

\section{Problem Formulation}

In order to explore the analytical results, we proceed to the study in a simple transit network as shown in Figure 1.

In Figure 1, node $a$ represents the residential zone generating $N$ commuters to the central business district (CBD) at node $c$. Suppose that at nodes $a$ and $b$, there are buses departing from these two nodes.
Under this setting, two bus lines, Line A and Line B, are designed to serve the demand $N$. In Figure 1, the chain dotted curve is Line A and the solid curve is Line B. Line A sends buses from $a$ to $c$ directly. Line B has a transfer stop at node $b$. The transfer is needed for users who choose Line B.

We assume that the percentage of oblivious users among all commuters is $k(0 \leq k \leq 1)$. Following the empirical study of Raveau et al. [20], we further assume that all oblivious users only choose Line A and other users are rational to choose one of the two lines according to the user equilibrium principle.

The total cost $C^{i}$ experienced by a commuter who travels from $a$ to $c$ by choosing Line $i, i=\{\mathrm{A}, \mathrm{B}\}$, can be formulated as

$$
C^{i}=P_{w} t_{w}^{i}+P_{v} t_{v}^{i}+P_{c}^{i}+p^{i},
$$

where $P_{w}$ and $P_{v}$ are the prices of waiting and in-vehicle times, respectively, $t_{w}^{i}$ and $t_{v}^{i}$ are the average waiting time at bus stops and in-vehicle time associated with Line $i$, respectively, $P_{c}^{i}$ is the body congestion cost occurring in bus carriage for Line $i$, and $p^{i}$ is the transit fare of Line $i$.

The body congestion cost is formulated by

$$
P_{c}^{i}= \begin{cases}0, & 0 \leq \tau \leq 1, \\ g(\tau), & \tau \geq 1,\end{cases}
$$

where $\tau$ is the passenger/capacity ratio. For obtaining the analytical results, we assume in this paper that $g(\tau)$ is a linearly increasing function of the ratio; that is, $g(\tau)=\theta \tau$. For $0 \leq \tau \leq 1$, all passengers can find seats and the body congestion does not exist, so $P_{c}^{i}=0$. In this paper, the capacity is simply defined as the total number of seats provided by a bus line.

For a constant arrival rate of passengers and regular bus headways, the average waiting time $t_{w}^{\mathrm{A}}$ for the commuters who choose Line A is

$$
t_{w}^{\mathrm{A}}=\frac{1}{2 f^{\mathrm{A}}},
$$

where $f^{\mathrm{A}}$ is the bus frequency of Line A.

Because the commuters who choose Line $B$ have to transfer at node $b$, their average waiting time is

$$
t_{w}^{\mathrm{B}}=\frac{1}{f^{\mathrm{B}}},
$$

where $f^{\mathrm{B}}$ is the bus frequency of Line $\mathrm{B}$.

The in-vehicle time includes the bus running time, the time waiting for other commuters' boarding at origin, and the time waiting for other commuters' alighting at destination. For different bus lines, the in-vehicle time is different.

For Line A, the in-vehicle time is

$$
t_{v}^{\mathrm{A}}=\frac{T_{0}^{\mathrm{A}}}{2}+\frac{t N^{\mathrm{A}}}{f^{\mathrm{A}}},
$$

where $T_{0}^{\mathrm{A}}$ is the bus cycle running time of Line $\mathrm{A}, t$ is the average boarding and alighting time for each commuter, and $N^{\mathrm{A}} / f^{\mathrm{A}}$ is the number of passengers boarding each bus of 
Line A. In (5), the first term of the right-hand side is the bus moving time and the second term is the boarding and alighting time caused by all passengers sequentially alighting and boarding.

For Line $\mathrm{B}$, there is a transfer at node $b$ and the in-vehicle time is

$$
t_{v}^{\mathrm{B}}=\frac{T_{0}^{\mathrm{B}}}{2}+\frac{2 t N^{\mathrm{B}}}{f^{\mathrm{B}}} .
$$

An equilibrium state is reached when all commuters are satisfied with their bus line choice. In other words, $k N$ oblivious users choose Line $\mathrm{A}$, and other rational users experience identical and minimal travel cost no matter they choose which line.

For facilitating the presentation of the essential idea, we assume that the passenger/capacity ratio of each line is larger than 1 and $P_{w}=P_{v}=P$. Based on these assumptions, the perfectly rational user equilibrium solution $\left(\bar{N}^{\mathrm{A}}, \bar{N}^{\mathrm{B}}\right)$, that is, when $C^{\mathrm{A}}=C^{\mathrm{B}}$ and $k=0$, is easily found. It follows that

$$
\begin{gathered}
\bar{N}^{\mathrm{A}}=\left(2 f^{\mathrm{A}} V^{\mathrm{A}} N\left(2 P t V^{\mathrm{B}}+\theta\right)-P V^{\mathrm{A}} V^{\mathrm{B}}\left(f^{\mathrm{B}}-2 f^{\mathrm{A}}\right)\right. \\
\left.+\left(p^{\mathrm{B}}-p^{\mathrm{A}}+P T_{0}^{\mathrm{B}}-P T_{0}^{\mathrm{A}}\right) f^{\mathrm{A}} V^{\mathrm{A}} f^{\mathrm{B}} V^{\mathrm{B}}\right) \\
\times\left(2 f^{\mathrm{B}} V^{\mathrm{B}}\left(P t V^{\mathrm{A}}+\theta\right)+2 f^{\mathrm{A}} V^{\mathrm{A}}\left(2 P t V^{\mathrm{B}}+\theta\right)\right)^{-1}, \\
\bar{N}^{\mathrm{B}}=N-\bar{N}^{\mathrm{A}},
\end{gathered}
$$

where $V^{i}$ is the bus capacity of Line $i, i=\{\mathrm{A}, \mathrm{B}\}$.

Clearly, the above bus line split solution is affected by the bus cycle running time, bus frequency, capacity, and fare. Next, we derive some results in three special cases.

Case $I\left(V^{\mathrm{A}}=V^{\mathrm{B}}=V, p^{\mathrm{A}}=p^{\mathrm{B}}=p\right.$, and $\left.f^{\mathrm{A}} \neq f^{\mathrm{B}}\right)$. Equation (7) becomes

$$
\begin{aligned}
& \bar{N}_{1}^{\mathrm{A}}=\left(2 f^{\mathrm{A}} N(2 P t V+\theta)-P V\left(f^{\mathrm{B}}-2 f^{\mathrm{A}}\right)\right. \\
&\left.+\left(P T_{0}^{\mathrm{B}}-P T_{0}^{\mathrm{A}}\right) f^{\mathrm{A}} f^{\mathrm{B}} V\right) \\
& \times\left(2 f^{\mathrm{B}}(P t V+\theta)+2 f^{\mathrm{A}}(2 P t V+\theta)\right)^{-1}, \\
& \bar{N}_{1}^{\mathrm{B}}=N-\bar{N}_{1}^{\mathrm{A}} .
\end{aligned}
$$

Case II $\left(f^{\mathrm{A}}=f^{\mathrm{B}}=f, p^{\mathrm{A}}=p^{\mathrm{B}}=p\right.$, and $\left.V^{\mathrm{A}} \neq V^{\mathrm{B}}\right)$. Equation (7) becomes

$$
\begin{gathered}
\bar{N}_{2}^{\mathrm{A}}=\frac{2 V^{\mathrm{A}} N\left(2 P t V^{\mathrm{B}}+\theta\right)+P\left(1+T_{0}^{\mathrm{B}}-T_{0}^{\mathrm{A}}\right) f V^{\mathrm{A}} V^{\mathrm{B}}}{2 V^{\mathrm{B}}\left(P t V^{\mathrm{A}}+\theta\right)+2 V^{\mathrm{A}}\left(2 P t V^{\mathrm{B}}+\theta\right)}, \\
\bar{N}_{2}^{\mathrm{B}}=N-\bar{N}_{2}^{\mathrm{A}} .
\end{gathered}
$$

Case III $\left(f^{\mathrm{A}}=f^{\mathrm{B}}=f, V^{\mathrm{A}}=V^{\mathrm{B}}=V\right.$, and $\left.p^{\mathrm{A}} \neq p^{\mathrm{B}}\right)$. Equation (7) becomes

$$
\begin{gathered}
\bar{N}_{3}^{\mathrm{A}}=\frac{2 N(2 P t V+\theta)+P V+\left(p^{\mathrm{B}}-p^{\mathrm{A}}+P T_{0}^{\mathrm{B}}-P T_{0}^{\mathrm{A}}\right) f V}{2(P t V+\theta)+2(2 P t V+\theta)} \\
\bar{N}_{3}^{\mathrm{B}}=N-\bar{N}_{3}^{\mathrm{A}} .
\end{gathered}
$$

When oblivious users are considered, that is, $k>0$, the following proposition can be obtained.

Proposition 1. If $k N \leq \bar{N}^{A}$, the oblivious users do not affect the equilibrium choice state; otherwise, the equilibrium choice state becomes $(k N,(1-k) N)$.

Proof. Substituting (2) to (6) into (1) yields

$$
\begin{aligned}
& C^{\mathrm{A}}=P \frac{1}{2 f^{\mathrm{A}}}+P\left(\frac{T_{0}^{\mathrm{A}}}{2}+\frac{t N^{\mathrm{A}}}{f^{\mathrm{A}}}\right)+\theta \frac{N^{\mathrm{A}}}{f^{\mathrm{A}} V^{\mathrm{A}}}+p^{\mathrm{A}}, \\
& C^{\mathrm{B}}=P \frac{1}{f^{\mathrm{B}}}+P\left(\frac{T_{0}^{\mathrm{B}}}{2}+\frac{2 t N^{\mathrm{B}}}{f^{\mathrm{B}}}\right)+\theta \frac{N^{\mathrm{B}}}{f^{\mathrm{B}} V^{\mathrm{B}}}+p^{\mathrm{B}} .
\end{aligned}
$$

Obviously, in (11) the total cost experienced by a commuter choosing Line A (or B) is linearly increasing with respect to the number of passengers choosing Line $A$ (or B).

Consider a corner of the initial state that all oblivious users choose Line A and all rest rational users choose Line B.

If $k N \leq \bar{N}^{\mathrm{A}}$, some rational users who have higher cost in Line B will switch to Line A until the equilibrium state $\left(\bar{N}^{\mathrm{A}}, \bar{N}^{\mathrm{B}}\right)$ is achieved.

If $k N>\bar{N}^{\mathrm{A}}$, the oblivious users who choose Line A are satisfied with their choice; meanwhile the rest rational users who choose Line B incur lower cost and will not change their choice. So the equilibrium state is $(k N,(1-k) N)$.

In this proposition, the parameter $k$ is crucial and can be calibrated by empirical survey (e.g., stated preference survey).

\section{Transit System Design}

In this section, we only consider the situation that $k N>\bar{N}^{\mathrm{A}}$ in which the equilibrium state $(k N,(1-k) N)$ is affected by oblivious users. In order to find the design variable, for example, frequency $f^{i}$, the total value of the resources (VRC) consumed by operators and users per hour has to be minimized as shown in (12). The first term on the right-hand side of (12) corresponds to the operation cost of fleet sizes times operational costs. The fleet of each line results from the product of frequency $f^{i}$ times its cycle time $t_{c}^{i}$. In the operational cost we consider a constant term $c_{0}$ and a variable term that grows with the size of the vehicle, $c_{1} V^{i}$. The second term on the right-hand side of (12) represents the total users' costs including the waiting costs, in-vehicle costs, and body congestion cost. Note that the total cost should not include the transit fare. We then have a minimization problem for each line as follows:

$$
\begin{array}{r}
\min \mathrm{VRC}^{i}=f^{i} t_{c}^{i}\left(c_{0}+c_{1} V^{i}\right)+\left(P_{w} t_{w}^{i}+P_{v} t_{v}^{i}+P_{c}^{i}\right) N^{i}, \\
i=\{\mathrm{A}, \mathrm{B}\},
\end{array}
$$


where $N^{\mathrm{A}}=k N$ and $N^{\mathrm{B}}=(1-k) N$. The cycle time $t_{c}^{i}$ is the summation of vehicle running time and standing time at stops. For Line $\mathrm{A}$, the running time is $T_{0}^{\mathrm{A}}$. The standing time is given by the delay caused by sequentially alighting and boarding time of a passenger $(t)$ times the number of passengers boarding each bus $\left(N^{\mathrm{A}} / f^{\mathrm{A}}\right)$. The cycle time for Line A can be written as

$$
t_{c}^{\mathrm{A}}=T_{0}^{\mathrm{A}}+\frac{t N^{\mathrm{A}}}{f^{\mathrm{A}}} .
$$

Similarly, the cycle time for Line B is

$$
t_{c}^{\mathrm{B}}=T_{0}^{\mathrm{B}}+\frac{2 t N^{\mathrm{B}}}{f^{\mathrm{B}}} .
$$

Solving the minimization problem (12), we have the following proposition.

Proposition 2. For Lines $A$ and $B$, suppose that the parameters $c_{0}, c_{1}, T_{0}^{A}, T_{0}^{B}, \theta$, and $t$ are constant and the vehicle size is determined by the demand; that is, $V=N / f$. Then, the optimal bus frequencies for each line, respectively, are

$$
\begin{gathered}
\bar{f}^{A}=\sqrt{\frac{2 t\left(c_{1}+P_{v}\right)(k N)^{2}+P_{w} k N}{2 T_{0}^{A} c_{0}}}, \\
\bar{f}^{B}=\sqrt{\frac{2 t\left(c_{1}+P_{v}\right)((1-k) N)^{2}+P_{w}(1-k) N}{T_{0}^{B} c_{0}} .}
\end{gathered}
$$

Proof. For Line A, the VRC can be written as a function of frequency only as follows:

$$
\mathrm{VRC}^{\mathrm{A}}=T_{0}^{\mathrm{A}} c_{0} f^{\mathrm{A}}+\frac{1}{f^{\mathrm{A}}}\left(0.5 P_{w} N^{\mathrm{A}}+t\left(P_{v}+c_{1}\right)\left(N^{\mathrm{A}}\right)^{2}\right)+M,
$$

where $M$ is a constant term, $M=\left(T_{0}^{\mathrm{A}} c_{1}+t c_{0}+0.5 T_{0}^{\mathrm{A}} P_{v}+\theta\right) N^{\mathrm{A}}$, and $N^{\mathrm{A}}=k N$.

Equation (17) shows that increasing frequency has a double effect. Taking a derivative of (17) with respect to frequency, we have

$$
\frac{\mathrm{dVRC}^{\mathrm{A}}}{\mathrm{d} f^{\mathrm{A}}}=T_{0}^{\mathrm{A}} c_{0}-\frac{1}{\left(f^{\mathrm{A}}\right)^{2}}\left(0.5 P_{w} N^{\mathrm{A}}+t\left(P_{v}+c_{1}\right)\left(N^{\mathrm{A}}\right)^{2}\right) .
$$

Making it equal to zero and noting that the second derivative is positive, we get the optimal frequency of Line A:

$$
\bar{f}^{\mathrm{A}}=\sqrt{\frac{2 t\left(c_{1}+P_{v}\right)(k N)^{2}+P_{w} k N}{2 T_{0}^{\mathrm{A}} c_{0}}} .
$$

Similarly, we can get the optimal bus frequency of Line B, as given by (16).

\section{Conclusions}

In this paper, we studied the equilibrium bus line choice behavior with oblivious users and investigated how the equilibriu$\mathrm{m}$ state is affected by these users. We further optimized each line's bus frequency of the transit system. Oblivious users are those who stubbornly adhere to some options, regardless of actual conditions. Obviously, such users or passengers indeed exist in reality. Hence, we have to consider them when formulating the option choice model. Our on-going work is to calibrate the model parameters and extend the proposed approach to explore the types of behavior in more complex transit networks.

\section{Conflict of Interests}

The authors declare that there is no conflict of interests regarding the publication of this paper.

\section{Acknowledgment}

The work described in this paper was supported by a Grant from the National Basic Research Program of China (2012CB725401).

\section{References}

[1] J. Wardrop, "Some theoretical aspects of road traffic research," in Proceedings of the Institution of Civil Engineers Part II, pp. 325378, 1952.

[2] C. F. Daganzo and Y. Sheffi, "On stochastic models of traffic assignment," Transportation Science, vol. 11, no. 3, pp. 253-274, 1977.

[3] Y. Sheffi, Urban Transportation Networks: Equilibrium Analysis With Mathematical Programming Methods, Prentice-Hall, Englewood Cliffs, NJ, USA, 1985.

[4] H. Yang and H. J. Huang, Mathematical and Economic Theory of Road Pricing, Elsevier, Oxford, UK, 2005.

[5] H. A. Simon, "A behavioral model of rational choice," The Quarterly Journal of Economics, vol. 69, no. 1, pp. 99-118, 1955.

[6] H. S. Mahmassani and G.-L. Chang, "On boundedly rational user equilibrium in transportation systems," Transportation Science, vol. 21, no. 2, pp. 89-99, 1987.

[7] J. Conlisk, "Why bounded rationality?" Journal of Economic Literature, vol. 34, no. 2, pp. 669-700, 1996.

[8] T.-Y. Hu and H. S. Mahmassani, "Day-to-day evolution of network flows under real-time information and reactive signal control," Transportation Research C, vol. 5, no. 1, pp. 51-68, 1997.

[9] H. S. Mahmassani and Y.-H. Liu, "Dynamics of commuting decision behaviour under Advanced Traveller Information Systems," Transportation Research C, vol. 7, no. 2-3, pp. 91-107, 1999.

[10] H. S. Mahmassani, “Trip timing," in Handbook of Transport Modeling, D. A. Hensher and K. J. Button, Eds., Elsevier, New York, NY, USA, 2000.

[11] Y. Lou, Y. Yin, and S. Lawphongpanich, "Robust congestion pricing under boundedly rational user equilibrium," Transportation Research B, vol. 44, no. 1, pp. 15-28, 2010.

[12] X. Di, H. Liu, J. Pang, and X. Ban, "Boundedly rational user equilibria (BRUE): mathematical formulation and solution sets," Transportation Research B, vol. 57, pp. 300-313, 2013. 
[13] G. Karakostas, T. Kim, A. Viglas, and H. Xia, "On the degradation of performance for traffic networks with oblivious users," Transportation Research B, vol. 45, no. 2, pp. 364-371, 2011.

[14] M. Kraus, "Discomfort externalities and marginal cost transit fares," Journal of Urban Economics, vol. 29, no. 2, pp. 249-259, 1991.

[15] W. H. K. Lam, C.-Y. Cheung, and C. F. Lam, "A study of crowding effects at the Hong Kong light rail transit stations," Transportation Research A, vol. 33, no. 5, pp. 401-415, 1999.

[16] H. J. Huang, Q. Tian, and Z. Y. Gao, "An equilibrium model in urban transit riding and fare polices," in Algorithmic Applications in Management, vol. 3521 of Lecture Notes in Computer Science, pp. 112-121, 2005.

[17] W. Vickrey, "Some implications of marginal cost pricing for public utilities," American Economic Review, vol. 45, no. 2, pp. 605620, 1955.

[18] H. Mohring, "Optimization and scale economies in urban bus transportation," American Economic Review, vol. 62, no. 4, pp. 591-604, 1972.

[19] S. R. Jara-Díaz, A. Gschwender, and M. Ortega, "Is public transport based on transfers optimal? A theoretical investigation," Transportation Research B, vol. 46, no. 7, pp. 808-816, 2012.

[20] S. Raveau, J. C. Muñoz, and L. de Grange, "A topological route choice model for metro," Transportation Research A, vol. 45, no. 2, pp. 138-147, 2011. 


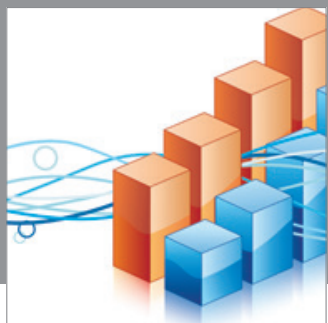

Advances in

Operations Research

mansans

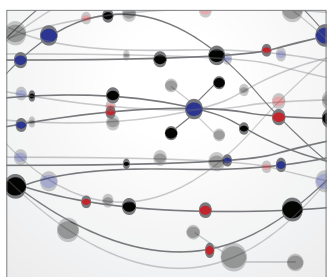

The Scientific World Journal
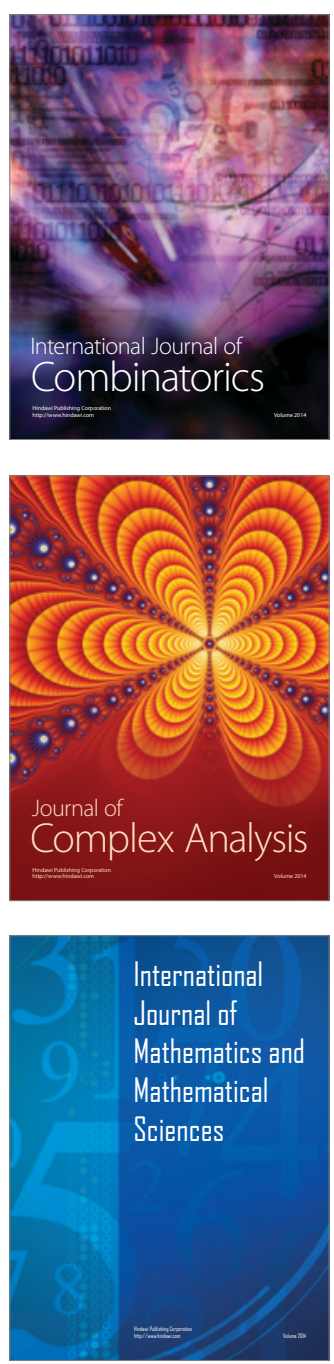
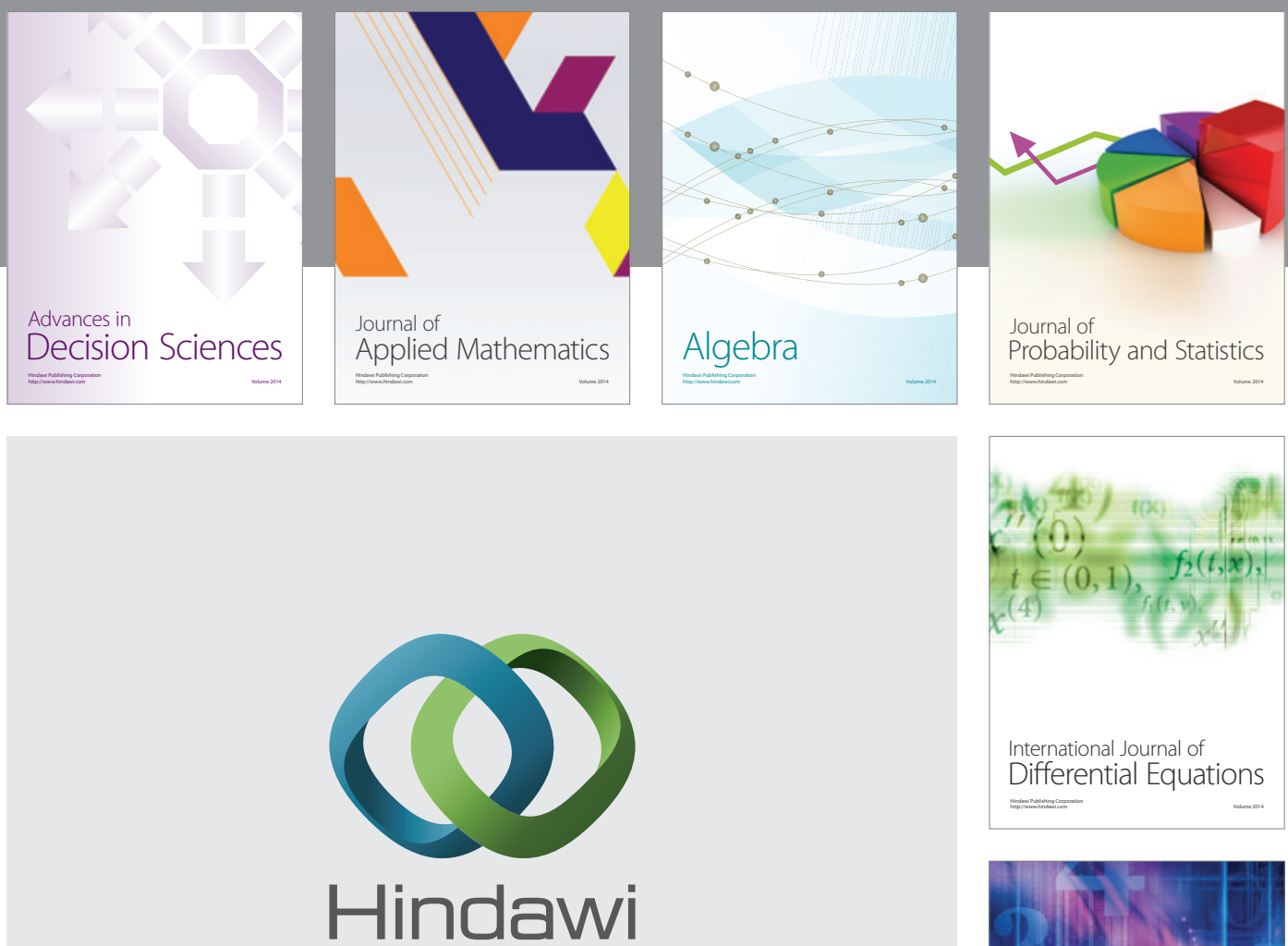

Submit your manuscripts at http://www.hindawi.com
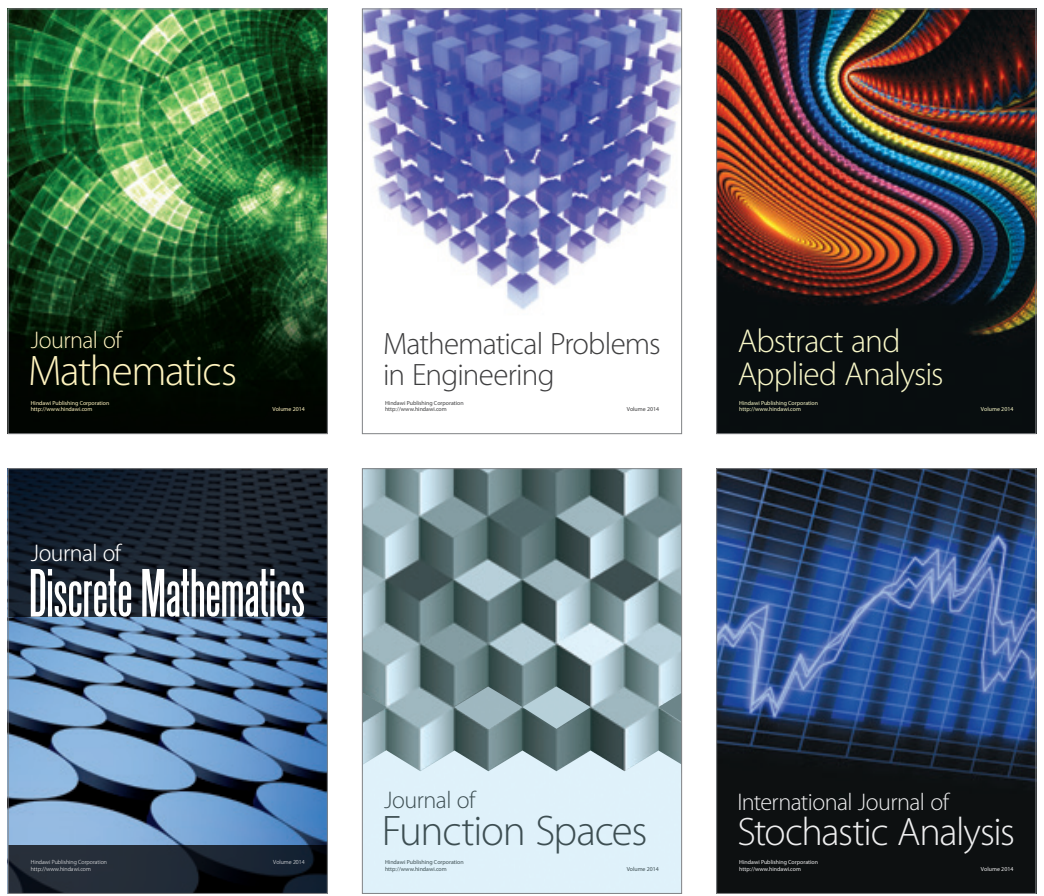

Journal of

Function Spaces

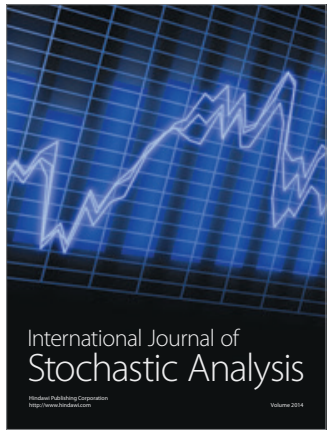

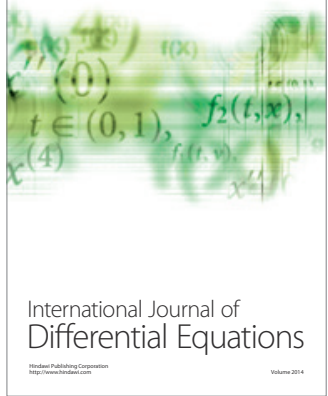
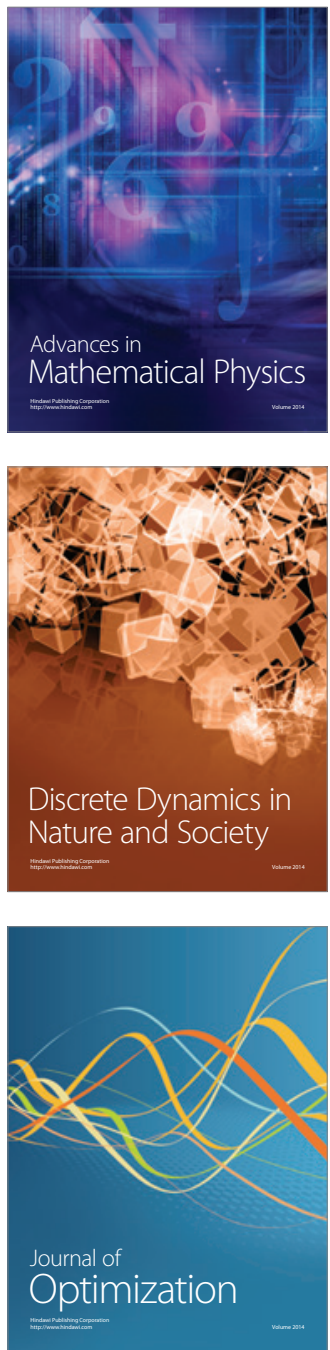